\title{
The Sleep Modeling in the Human Organism
}

Janos Vincze*, Gabriella Vincze-Tiszay

Health Human International Environment Foundation, Budapest, Hungary

*Corresponding Author: Janos Vincze, Health Human International Environment Foundation, Budapest, Hungary

Received date: April 19, 2021; Accepted date: May 07, 2021; Published date: May 10, 2021

Citation: J Vincze, G Vincze-Tiszay. (2021) The Sleep Modeling in the Human Organism. Clinical Research and Clinical Trials. 3(4); DOI: 10.31579/2693-4779/039

Copyright: (C) 2021 Janos Vincze. This is an open access article distributed under the Creative Commons Attribution License, which permits unrestricted use, distribution, and reproduction in any medium, provided the original work is properly cited.

\begin{abstract}
There are three alternating states of vigilance throughout our lives: wakefulness, NREM, and REM sleep. We usually yawn before falling asleep. Yawning is an ancient reaction, an instinctive action, manifested in a person by drowsiness or boredom. Yawning is often associated with the need for stretching. Yawning is a less strong territorial reflex. During deep sleep muscular tone is sharply reduced. Relaxation of the muscles and the lowering of their tone, howeever, are not constant and necessary components of sleep. Analysis of EEG recordings soon revealed that sleep is by no means a uniform process, but can be divided into at least two sharply separated states: one is characterized by slow waves in the EEG that are completely separate from the activity of wakefulness: this socalled slow wave sleep; the other is the so-called paradoxical sleep. Hypnopedia, as a discipline, deals with the input of fixed information introduced during the period of natural sleep, also known as sleep learning. Our hypnopedia researches was a pleasant surprise, because they were able to reproduce texts they did not know with an efficiency of approx. $25 \%$.
\end{abstract}

Key words: sleep; biophysical modeling; EEG; hypnopedia

\section{Introduction}

Every science has central issues, such a key problem for biophysics is modeling. The phenomena that take place in the world around us are usually very complex. In living systems, many parameters are present at the same time in almost every process, and they are intricately interdependent. There are hardly any cases where we can expect only a few and such simply interrelated parameters in relation to which we can use simple models in their examination and description. At first, we often think that the difficulty comes from the large number of parameters, but later we realize that we need to look deeper for the cause, namely in the nonlinear context of each parameter.

Therefore, we select the most characteristic parameters of the processes and create a construction in which the processes can be implemented through the processes with an adequate approach. A subset of our existing knowledge and assumptions, as well as the subset to be examined selected from the set of experiments performed (there may be thought experiments!) and the results of the examinations are called models.

Thus, the model cannot be identified with the system under examination, but it facilitates a more complete understanding of it. Modeling, in its simplest form, is a sharp, polarized question about the phenomenon in question. The model is in a similarity relation to the modeled system. A model is a theoretically or materially realized system that, by reflecting or reproducing an object of research, is able to temporarily replace it. The model can always be interpreted only in conjunction with the modeling.
Two systems (A and B) are considered to be models (or modeled) relative to each other if it is possible to homomorphically map system A to system $\mathrm{A}^{\prime}$ and system B to system B' so that A' and B' are isomorphic to each other. Thus, the model is a simplified, comprehensible, practically implemented or theoretically imagined, proportionally reduced or enlarged, most of the time accurately describable in terms of mathematics, idealized copy of the system examined, which more or less correctly illustrates the peculiarities of the system or process examined.

When creating a model, one must be aware that the model is not the same as the system under study and does not fully reflect all its properties. Nevertheless, the correctly formed model bears the most important criteria of the examined system and is thus suitable for the exploration of the decisive regularities.

A lucky model describes the knowledge of a given moment, but in addition it allows assumptions, and these can be translated into the language of reality and model specific situations. A well-chosen model helps not only to raise the issue, but also to study the phenomena scientifically. Biophysics cannot lack models, but it needs to correspond to actual reality. Not everything can be modeled.

\section{The Sleep}

The significant time spent sleeping also indicates that it is a matter of meeting some important biological need, as evidenced by the fact that if we prevent sleep for days, the body then makes up for the omission, and even completes paradoxes with particular precision. The evolution of the 


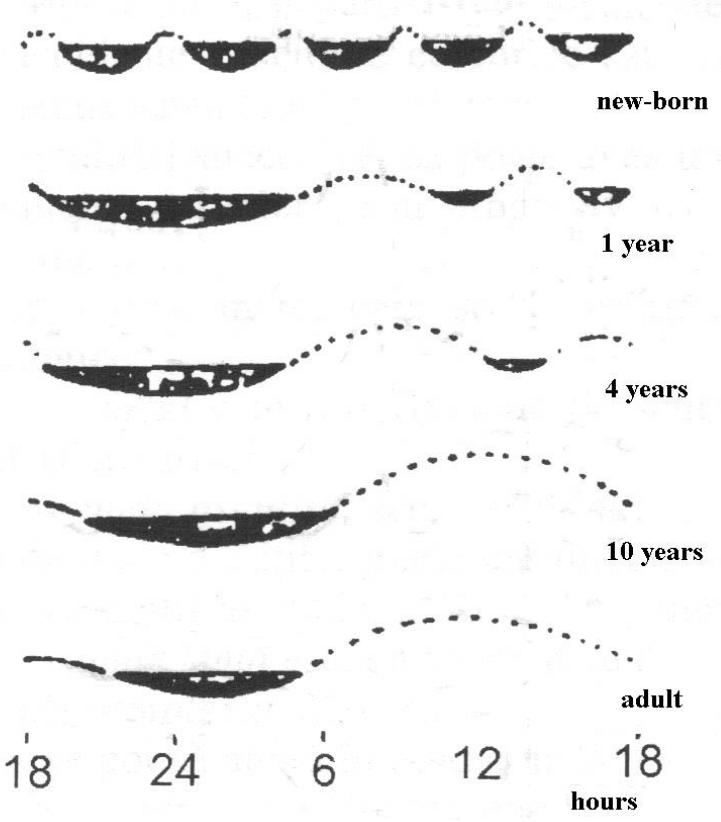

Figure 1: States of vigilance (wakefulness, sleeping) in the ontogenesis.

During deep sleep muscular tone is sharply reduced. Most muscles in a sleeping person are completely relaxed, which is why objects fall from the hands of a person sleeping in a sitting position and why his head sinks onto his chest, and his trunk sags.

Relaxation of the muscles and the lowering of their tone, howeever, are not constant and necessary components of sleep. Even during normal sleep various movements are possible, and a definite pose can be maintained for a long time, as when sleeping in a sitting posture. All the senses, i.e. vision, hearing, taste, smell, and the tactile sense are greatly reduced, and a much stronger stimulus is required to induce a reaction in a sleeping person than when he is awake.

The reflex function is sharply reduced during sleep. Conditioned reflexes are inhibited, unconditioned ones are considerably weakened. Their thresholds of stimulation are considerably raised, and the latent period lengthened. Sensomotor changes dominate the picture of sleep as compared with changes in vegetative functions.

The significant time spent sleeping also indicates that it is a matter of meeting some important biological need, as evidenced by the fact that if we prevent sleep for days, the body then makes up for the omission, and even completes paradoxes with particular precision.

Common sense dictates that sleep is simply perceived as rest, regeneration. However, it is easy to see that the body does not need sleep to rest. Is it about resting the brain? However, the regeneration of nerve cells is a very quick process, it is believed that the neurons controlling the heartbeat or breathing rest during the interval between two consecutive breaths or heartbeats.

\section{Electroencephalogram}

The possibility of objective experimental studies regarding sleep was provided only by the development of technology in the 1950s. This was mainly due to the widespread use of electroencephalographic examinations. An electroencephalogram (EEG) is a graphical representation of the electrical activity of brain neurons; a measurement signal on the order of microvolts that shows the change in activity of thousands of neurons over time. The EEG is registered with low-resistance electrodes placed on the scalp.

EEG diversion is performed using low-resistance electrodes placed on the scalp. Depending on the equipment, diversion can take place on more than 200 channels with a defined measurement interval. The principle of measurement is as follows: we measure the potential difference between two electrodes. Bipolar conduction between active electrodes (below which there are active neurons) or monopolar conduction between inactive (reference) electrodes. The derived signal is amplified and then filtered according to the frequency range to be examined. The result obtained can be used to perform various analyzes on the given signals with the help of computers - quantitatively by fast Fourier analysis.

Electrodes placed on the scalp do not conduct the action potential, but synaptic potentials because the synaptic voltage is lower (in the order of microvolts) than the action potential $(1-2 \mathrm{mV})$. The action potential is measured with microelectrodes due to its speed, while the synaptic potential can be measured with a cap placed on the scalp. AP has a nature of all or nothing, while in the case of synaptic potentials, their mathematical sum is derived in the form of EEG, i.e. the sum of voltage fluctuations.

\begin{tabular}{|l|l|l|}
\hline EEG waves & Frequency $(\mathbf{H z})$ & Tension $(\mu \mathbf{V})$ \\
\hline Delta $(\delta)$ & $0,5-3,5$ & $100-200$ \\
\hline Theta $(\theta)$ & $4-7,5$ & $<30$ \\
\hline Alfa $(\alpha)$ & $8-12$ & $30-50$ \\
\hline Beta $(\beta)$ & $13-30$ & $<20$ \\
\hline Gamma $(\gamma)$ & $30-75$ & $<10$ \\
\hline
\end{tabular}


Analysis of EEG recordings soon revealed that sleep is by no means a uniform process, but can be divided into at least two sharply separated states: one is characterized by slow waves in the EEG that are completely separate from the activity of wakefulness: this so-called slow wave sleep; the other is the so-called paradoxical sleep, in which wake-like activity is seen on the EEG, although the individual's sleep is deepest at this time hence the contradictory paradoxical marker (REM - Rapid Eye Movement).

\section{Paradoxical sleeping}

Slow-wave sleep, as its name implies, is caused by slow waves occurring in increasing numbers and at lower and lower frequencies in the EEG, as well as periods of 2-3 seconds consisting of more frequent waves, being characterized by the so-called "sleep spindles". Based on the electrical activity of the brain, several stages of slow-wave sleep can be distinguished, e.g. four sleep states are usually defined from EEG curves, denoted by numbers 1-4 from superficial sleep to deep sleep. Although the different origins of each stage arise from time to time, slow-wave sleep is mostly considered qualitatively one, with each stage denoting different sleep depths.

Characteristics of each phase:

1. NREM phase: forms a transition between wakefulness and sleep, the feeling of drowsiness increases. Brain waves and muscle activity slow down.

2. NREM phase: a light sleep period during which eye movement stops, brain waves slow further, heart rate and body temperature decrease.

3. NREM phase: characterized by extremely slow brain waves. Blood pressure drops and breathing slows down.

4. NREM phase: the phase of the deepest sleep, the brain produces the slowest waves. There is no eye movement, but the limb muscles are still able to move. Waking up someone is the most difficult at this stage. Phases 3 and 4 are essential for restoring the body's energy, and this is when growth hormones are released as well.

5. REM phase: characterized by strong brain activity, this is when we dream. The motor muscles are temporarily paralyzed.

Under normal conditions, electrical activity in the brain follows the development of sleep well. Sleep progresses from stage 1, i.e., from the most superficial stage, to stage 4, then its depth decreases again, and before another stage 1 occurs - paradoxical sleep appears. Sleep is deepest in the first half of the night, especially in the first sleep cycle. In later cycles, the amount of stage 4 is less and less, towards the morning the slow-wave sleep does not even go beyond stage 2 .

Paradoxical sleep is a qualitatively different state from slow-wave sleep. Cerebral electrical activity is characterized by vigorous, rapid activity, which can sometimes exceed wakefulness activity. The muscles relax completely. Sometimes, however, stormy movements occur, but they are always limited to a specific group of muscles: the wrist moves, one finger bends, and so on. Occasionally, high-amplitude eye movements are very common and are often accompanied by blood filling of the genitals, resulting in erection of the penis or clitoris in women. The heartbeat suddenly increases and then decreases, blood pressure fluctuates sharply, breathing may become irregular, and even temporary complete respiratory arrest may occur. Respiratory arrest in infants can sometimes be fatal. During paradoxical sleep, the thermoregulation is switched off, but since the length of each paradoxical phase is 6-15 minutes, no significant cooling can occur on the covered person. The amount of paradoxical sleep is highest in newborns, reaching up to $70 \%$ of total sleep time. This drops to about $20 \%$ in adulthood.

\section{A hypnopedic research}

Hypnopedia, as a discipline, deals with the input of fixed information introduced during the period of natural sleep, also known as sleep learning. In the different phases of sleep, the perception of stimuli is preserved. For example, a story, a tale, whispered in the ear of a sleeping person, becomes "familiar" after awakening, without being able to tell its content, or when and from whom, where it was heard.

In the early 1960s, we set up a sleep laboratory at the Institute of Biophysics, University of Medicine. Compared to the circumstances at the time, it could be said to be modern: EEG, EMG, portable ECG, tape recorders, oscilloscopes and much more that we would have needed, in addition to these, were put together by us. There were plenty of experimental subjects because it was natural for medical students to perform various human experiments on volunteers.

I applied first to have a "test drive" done by my colleagues. They taught me a text while sleeping that I had no idea about. After multiple team discussions we have decided to register as many parameters during sleep as possible. This is how we realized that when eye movement displays the REM phase, the subjects do not learn, as the sleeping organism wakes up easily, and if this occurs several times within a given period, they would feel very tired during the daytime. A similar interpretation was given to the importance of breathing, the alternation of the EEG waveforms and the warning signs of muscle movement.

After several months of experimentation, we signed up with eight medical students for the institutional meeting. Colleagues working in the institute received the full text of a poem, along with the author's name. The head of the institute asked whether the medical students knew the poems, whether they heard them or not, and then he read the first verse. All eight students said that they were not familiar with the poem. Then we started the recording from the second verse that they learned during sleep. The students were able to recite the poem with an efficiency of approx. $75 \%$. It was an incredible and spectacular success and evidence available for everyone regarding learning during sleep. The result: the laboratory was shut down by the military.

Now, at an old age, I repeated the experiment again under special family circumstances, without any means. I spent more time with my kids and agreed with them to repeat my hypnopedia experiments on my grandchildren. On several occasions, I performed the following experiment on 10 of my grandchildren - between 6 and 10 years old. After they fell asleep I went into their room and repeated a story I had written 10 times for an hour for 7 days. So my grandchildren, while sleeping, heard the same text 70 times as I read it. We later checked to see if they could repeat or recall anything from the text - completely unknown to them - that was read to them at night. The result was a pleasant surprise, because they were able to reproduce texts they did not know with an efficiency of approx. $25 \%$.

\section{Modeling}

We assume that during hypnopedia we want to convey certain information to the given person and later we want to check its recording, that is, we have to ask back, so it can be considered a dialogue.

Therefore, it is worth writing the information matrix before starting hypnopedia, because with the help of this we break down the information itself into very small parts and in this way the essential and less important parts of the information duration become more and more obvious to the experimenter. 
Let our information matrix be:

$$
B_{i}=\left\|\begin{array}{|llll}
B_{11} & B_{12} & \cdots & B_{1 k} \\
B_{21} & B_{22} & \cdots & B_{2 k} \\
\vdots & \vdots & \vdots & \vdots \\
B_{p 1} & B_{p 2} & \cdots & B_{p k}
\end{array}\right\|
$$

During the experiment, the information was repeated $\mathbf{m}$ times. The question immediately arises: how many times should it be repeated in a single experiment? We found that the information return efficiency $\left(\eta_{\mathrm{d}}\right)$ is maximal when in an experiment the number of repetitions is of approx. 5-7 times $\left(\mathrm{m}_{1}\right)$.

$$
\eta_{d}=\frac{B_{i}^{m_{i}}}{B_{i}}
$$

Efficacy could be increased if the experiment was repeated during several consecutive sleeps:

$\mathrm{m}_{1}+\mathrm{m}_{2}+\ldots+\mathrm{m}_{5} \approx 35$

Thus, maximum efficiency $\left(\eta_{\mathrm{d}} *\right)$ is reached when we repeated the information we wanted to enter about 35 times on 5 occassions:

$$
\eta_{d}^{*}=\frac{B_{i}^{m_{1}+m_{2}+\cdots+m_{5}}}{B_{i}}
$$

We analyzed certain elements of the $\mathbf{B}_{\mathbf{k d}}$ information matrix according to the importance and severity of the information. In the acquisition of wakefulness information, the subjects selectively memorized the same information, i.e. transferred it to their preliminary information pyramid, compared it with their previous knowledge, and then recorded it. In the case of recording during sleep, there is a lack of comparison, and therefore the essential and much less important parts are recorded with the same efficiency by human memory, the maximum efficiency of reproduction is usually between 25 and 30 percent.

The learning technique of sleep learning is limited to repetitions, thus consolidating those learned because the learning of new knowledge takes place in an alert state. This does not preclude the recording of new information during sleep learning, which is recommended in such cases e.g. learning foreign words, geographical names, historical dates, so simple memorizable data.

\section{References}

1. Vincze, J. (2009) Biophysics of the Sleep. NDP P, Budapest.

2. Vincze, J. (2010) Envirimental Biophysics. NDP P., Budapest.

3. Vincze, J. (2015) The Capital Chapter of Biophysics. $5^{\text {th }}$. Ed. NDP P., Budapest.

4. Rasmussen, B.K, Jensen, R, Schroll, M. (1992) Interrelation between Sleep Symtons and their Treatment. Arch. Neurol; 49: 914-920.

5. Vincze, J. (1984) The Cross-effect in the Living Systems. Med. P. Budapest.

6. Cornelissen, G., Otsuka, K. (2017) Chronobiology of aging. Gerontology; 63(29 118-128.

7. Rishikesh, M. Agarwal, S.K. (2019) Evaluation of obstructive sleep apnea in patients with chronic obstructive pulmonary disease. Intern. J. Current Advanced Res; 7(1) 9342-9346.

8. Vincze, J. (2007) Interdisciplinarity, NDP P., Budapest.

9. Vincze, J. (2018) Medical Biophysics. NDP P., Budapest.

10. Scullin, M., K., Bliwise D. L. (2015) Sleep, Cognition, and Normal Aging: Integrating a Half Century of Multidisciplinary Research. Psychol. Science; 10:97-137.

11. Vincze, J. (2020) Biophysics of the Apparatuses. NDP P. Budapest.

12. Sarson, E, Cobelli, C. (2014) Modelling Methodology for Physiology and Medicine. Elsevier.

13. Vincze, J. (2020) The Biophysical Modeling of the Apparatuses in the Human Organism. Lambert Academic Publishing, Berlin.

14. Vincze J, Vincze-Tiszay, G. (2021) The Theories of the Sleep Regulation in the Human Organism. Technium; 3(1)138-141.

15. Vincze J. (2021) Biophysical vademecum. I. NDP P. Budapest

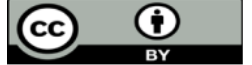

This work is licensed under Creative Commons Attribution 4.0 License

To Submit Your Article Click Here: Submit Manuscript
Ready to submit your research? Choose Auctores and benefit from:

*ast, convenient online submission
*igorous peer review by experienced research in your field
*apid publication on acceptance
* authors retain copyrights
* imique DOI for all articles
immediate, unrestricted online access

At Auctores, research is always in progress.

Learn more www.auctoresonline.org/journals/clinical-research-andclinical-trials- 